\title{
On Proper Specification in Tourism Research
}

\author{
A.George Assaf \\ Associate Professor \\ Isenberg School of Management \\ University of Massachusetts-Amherst \\ Email :assaf@isenberg.umass.edu
}

Mike Tsionas

Professor of Econometrics

Lancaster University Management School

Email:m.tsionas@lancaster.ac.uk

\section{Introduction}

Despite the excessive use of regression modelling in the field, it is surprising that not much attention has been given to some critical issues that affect the process of hypothesis testing. In this note, we argue that extensive diagnostic testing of the model is required before proceeding with hypothesis testing or other usages of the model. Problems like autocorrelation and heteroscedasticity should be interpreted as misspecification tests rather than as problems that merely affect standard errors (which is the case only in correctly specified models). Moreover, we suggest out-of-sample forecasting as a tool for model validation.

To illustrate, we use a simple regression model with an interaction term. Often, we test hypotheses about moderating variables using the following:

$$
y=\beta_{0}+\beta_{1} x_{1}+\beta_{2} x_{2}+\beta_{3} x_{1} x_{2}+u .
$$

For instance, when $\beta_{3} \neq 0$ we conclude that the moderating impact is significant.

Prior to making such conclusions, it is important to go through several and necessary important steps that are often not reported in tourism papers and may significantly affect the results.

1. First, the issue of specification: there is, in fact, no guarantee that the functional form in (1) is acceptable (or "true"). While such a model may produce "reasonable" results, this may be misleading because of misspecification problems. Misspecification may be detected using diagnostic tests (RESET, heteroscedasticity, and autocorrelation). Such diagnostics tests are, fortunately, sensitive in various forms of misspecification. Reporting heteroscedasticity tests before 
testing hypotheses (such as $\beta_{3}=0$ in (1)) ${ }^{1}$ is important. For instance, models such as (1) may fail several diagnostics because of misspecification problems.

2. Second, the issue of reverse causality, which occurs when $x_{1}, x_{2}$ and $y$ are jointly determined, leading to endogeneity problems that may affect adversely the issue of estimating models like (1).

3. Third, and related to point 1 above, we rarely see nonparametric models like $y=f\left(x_{1}, x_{2}\right)$ being estimated.

We emphasize and discuss the above in more detail and illustrate using a tourism application. We also introduce and examine the performance of specification tests for panel data which, to our knowledge is novel. The baseline model is a regression where "sales" depends on advertising, size and the interaction of advertising and size. Although the reported results are sensible, we look deeper into the problem and uncover problems with the specification. The model is misspecified in terms of the functional form based on the RESET test. In turn, this motivates us to investigate two other models; a model with fixed effects and a nonparametric model. The fixed-effects model also fails the RESET test. Although the nonparametric model passes the RESET test it fails a heteroscedasticity test: If heteroscedasticity is not merely an issue that requires light-hearted application of robust standard errors (such as HAC) but is, instead, indicative of specification error (omitted variables for example) then we have a warning that the nonparametric model is misspecified as well. This motivates us to investigate a dynamic models and reverse causality issues. A dynamic panel data model passes both RESET and heteroscedasticity tests and, therefore, can be considered as a valid working platform for further analysis.

\section{How to Proceed?}

Multiple regression models are prone to functional form misspecification. The specified model does not always account properly for the relationship between the outcome and observed variables. For instance, there is no reason to assume that a relationship is linear. Omitting a quadratic term or an interaction term when they should be included, may lead to functional form misspecification or omitted variable bias.

Specification should be the first and most important critical step ${ }^{2}$. Tests such a Ramsey's RESET are designed to detect nonlinearities in the model (arising from possibly excluding powers of the existing regressors) but not omitted variables per se. Misspecification will often result in beteroscedasticity and / or autocorrelation. Reverse causality should be also tested when appropriate. One can also use non-parametric

\footnotetext{
${ }^{1}$ In other instances, we may be interested in testing economic restrictions like $\beta_{1}+\beta_{2}=1$, inequality restrictions etc.

${ }^{2}$ In general, the specification of regression models should be driven by theoretical considerations, but we can never pretend that we have "too much theory".
} 
regression to avoid imposing restrictive a priori specifications on the functional form. However, there is no guarantee that the non-parametric regression will pass all these tests if there are omitted variables. We discuss below all these issues in more detail using a real example.

\section{Data and Results}

We use a well-known application on the relationship between advertising and sales. We use data on 21 US hotels and restaurants covering a balanced sample of publicly traded companies from 2001 to $2012(21 * 10=210$ observations). We propose the following models for comparison:

$\ln$ sales $=\alpha_{0}+\alpha_{1} A d v+\alpha_{2} A d v^{2}+\alpha_{3}$ Size $+\alpha_{4} A d v *$ Size $+\alpha_{5}$ Leverage $+\varepsilon$

$\ln$ sales $=\alpha_{0}+\alpha_{1} A d v+\alpha_{2} A d v^{2}+\alpha_{3} A d v^{3}+\alpha_{4} \operatorname{Size}+\alpha_{5} \operatorname{Siz} e^{2}+\alpha_{6} A d v^{*}$ Size $+\alpha_{7}$ Leverage $+\varepsilon$ (Model 2)

where sales is total sales ${ }^{3}, A d v$ is advertising expenses, Size is hotel size, Leverage is financial leverage and is used as a control variable. In line with the previous literature that suggests an Sshaped relationship between advertising and sales, we include both the square and cubic term of advertising (Johansson, 1979) in Model 2.

As we have panel data, we tried estimating the models using fixed effects in a static and dynamic framework:

$$
\begin{gathered}
y_{i t}=\alpha_{i}+x_{i t}^{\prime} \beta+u_{i t}, \\
y_{i t}=\alpha_{i}+\rho y_{i, t-1}+x_{i t}^{\prime} \beta+u_{i t} .
\end{gathered}
$$

For comparison we also estimated the following model in a non-parametric fashion: $\ln$ sales $=f(A d v$, Size, Leverage $)$, but as can we see from Table 1, the non-parametric model failed the heteroscedasticity test $\left(\mathrm{H}_{\mathrm{o}}\right.$ : Homoscedasticity). The fixed effect estimation also fails one of the two tests in either model. The dynamic specification (using the estimator of Arellano and Bond, 1991), using two lags for ln sales), on the other hand, passes both tests for Model $2^{4}$.

The results confirm that sales in Model 2 should be modeled using a dynamic specification, supporting other related studies in the area (Assaf et al. 2012). Along with the specification tests, we also tested for reverse causality between sales and advertising using the Dumitrescu-Hurlin (2012) test but we did not find any reverse causality problem $(p \text {-value }=0.9133)^{5}$. For additional evidence, we also tested for reverse causality using the following DPD model:

${ }^{3}$ We used the $\log$ of sales due to the highly skewed nature of this variable. We also tried "sales" as a dependent variable but the model failed all specification tests.

${ }^{4}$ For panel data, we constructed the RESET and heteroscedasticity tests manually. The regressors of these tests are the variables in Model 2, their squares and interactions. The dependent variables are the residuals and the logarithm of absolute residuals, respectively (we provide in Appendix B more validation about the performance of these tests). We also tested for autocorrelation in STATA and we did not find an autocorrelation problem. The static model, on the other hand, failed the autocorrelation test.

${ }^{5}$ Previous studies have also found evidence of one-way causality from advertising to sales (Kim, 2007). 


$$
\begin{aligned}
& y_{i t}=\alpha_{i}+\beta_{11} y_{i, t-1}+\beta_{12} x_{i, t-1}+u_{i t}, \\
& x_{i t}=\lambda_{i}+\beta_{21} y_{i, t-1}+\beta_{22} x_{i, t-1}+v_{i t},
\end{aligned}
$$

where $\alpha_{i}$ and $\lambda_{i}$ represent fixed effects, and $u_{i t}, v_{i t}$ are only contemporaneously correlated. We have reverse causality when $\beta_{21} \neq 0$. Of course, more lags can be included and weakly exogenous regressors may be present in this specification. This model is a panel vector autoregression (PVAR) and, in fact, can serve as a prototype for several empirical applications to control for both dynamics as well as reverse causality ${ }^{6}$. We tried the model in (3) using all variables in Model 2, but again, we failed to find evidence in favor of reverse causality between sales and advertising.

To further test the performance of our dynamic specification, we examined whether it is acceptable (leaving the notions of "correct" and "true" behind) in out-of-sample forecasting. We believe that any unacceptable model is very likely to fail this exercise. We estimated the model for the first seven years of our sample, and we forecast out-of-sample for the remaining three years. The results are shown in Figure 1, where it is clear that the deviations between actual and predicted values are, apparently, small and randomly scattered around the $45^{\circ}$ line ${ }^{7}$.

Parameter estimates for the dynamic specification are reported in Table 2. In Figure 2, we also report the elasticity of advertising across our whole sample. The average elasticity is around 0.36 , indicating that a 10\% increase in advertising leads to around 3.6\% increase in sales. Such impact seems to be reasonably substantial, although we recommend deriving the standard errors of these elasticity for a more concrete evidence of the impact of advertising on sales. Obviously, one method to derive those standard errors is the delta method.

\section{Concluding Remarks}

In this note, we highlighted the importance of testing for specification before proceeding with further regression analysis. Often in tourism research we report the results from a single model without first confirming that the functional form and related diagnostics ${ }^{8}$ of such model are acceptable.

The message from our Monte Carlo simulations is that standard diagnostic tests and simple modifications when we have panel data, can reveal problematic aspects of the data. For example, autocorrelation and heteroscedasticity diagnostics are sensitive to various forms of misspecification

\footnotetext{
${ }^{6}$ Of course, the issues of missing variables and correct functional form are still at large with this model, so extensive diagnostic testing is required to validate the model.

${ }^{7}$ As a benchmark example, we provide in Appendix A an example of a model that fails the out- of- sample forecasting. This model is based only on a simple linear relationship between log of sales and the log of the following covariates: advertising, size and leverage.

${ }^{8}$ By "diagnostics" we refer always to heteroscedasticity, autocorrelation, and RESET tests.
} 
and the same is true for the RESET test -although it needs a simple modification in the case of panel data.

Although there is some confusion over specification tests versus residual diagnostics, we should point out that, for the most part, specification tests are residual diagnostics and, fortunately, such diagnostics are sensitive to specification problems. If a model does not pass the diagnostics we presented, then it cannot be used. If it does, then it can be subjected to further scrutiny. Our recommendation is to perform diagnostic testing, and subject the model to out-of-sample tests such as the one in Figure 1.

Table 1- Heteroscedasticity and RESET Tests

\begin{tabular}{|l|c|c|}
\hline & Heteroscedasticity Test & RESET Test \\
\hline $\begin{array}{l}\text { Non- } \\
\text { Parametric } \\
\text { Regression }\end{array}$ & p-value $=0.00$ & -value $=0.35$ \\
\hline $\begin{array}{l}\text { Panel } \\
\text { Regression- }\end{array}$ & p-value $=0.65$ (Model 1$)$ & p-value $=0.00$ (Model 1) \\
Effect & p-value $=0.06$ (Model 2) & p-value $=0.04$ (Model 2) \\
\hline $\begin{array}{l}\text { Panel } \\
\text { Regression- } \\
\text { Dynamic }\end{array}$ & p-value $=0.00($ Model 1) & p-value $=0.80($ Model 1) \\
\hline
\end{tabular}

Table 2. Parameter Estimates-Dynamic model

\begin{tabular}{|l|c|c|c|}
\hline & Coefficient & $\mathrm{Z}$ & $\mathrm{P}>|\mathrm{z}|$ \\
\hline Ln Sales (t-1) & 0.465 & 2.98 & 0.003 \\
\hline Ln Sales (t-2) & -0.089 & -1.33 & 0.184 \\
\hline Adv & 0.0087 & 3.10 & 0.002 \\
\hline Adv2 & $-4.07 * 10^{-6}$ & -0.54 & 0.590 \\
\hline Adv3 & $2.39 * 10^{-9}$ & 0.44 & 0.663 \\
\hline Size & -1.0759 & -1.31 & 0.191 \\
\hline Size2 & 0.3016 & 2.25 & 0.025 \\
\hline Adv*Size & -0.0014 & -1.96 & 0.050 \\
\hline Leverage & 0.1138 & 1.57 & 0.116 \\
\hline _cons & 4.6049 & 4.31 & 0.000 \\
\hline
\end{tabular}


Figure 1. Comparison of actual and fitted values in out-of-sample validation -Dynamic Model

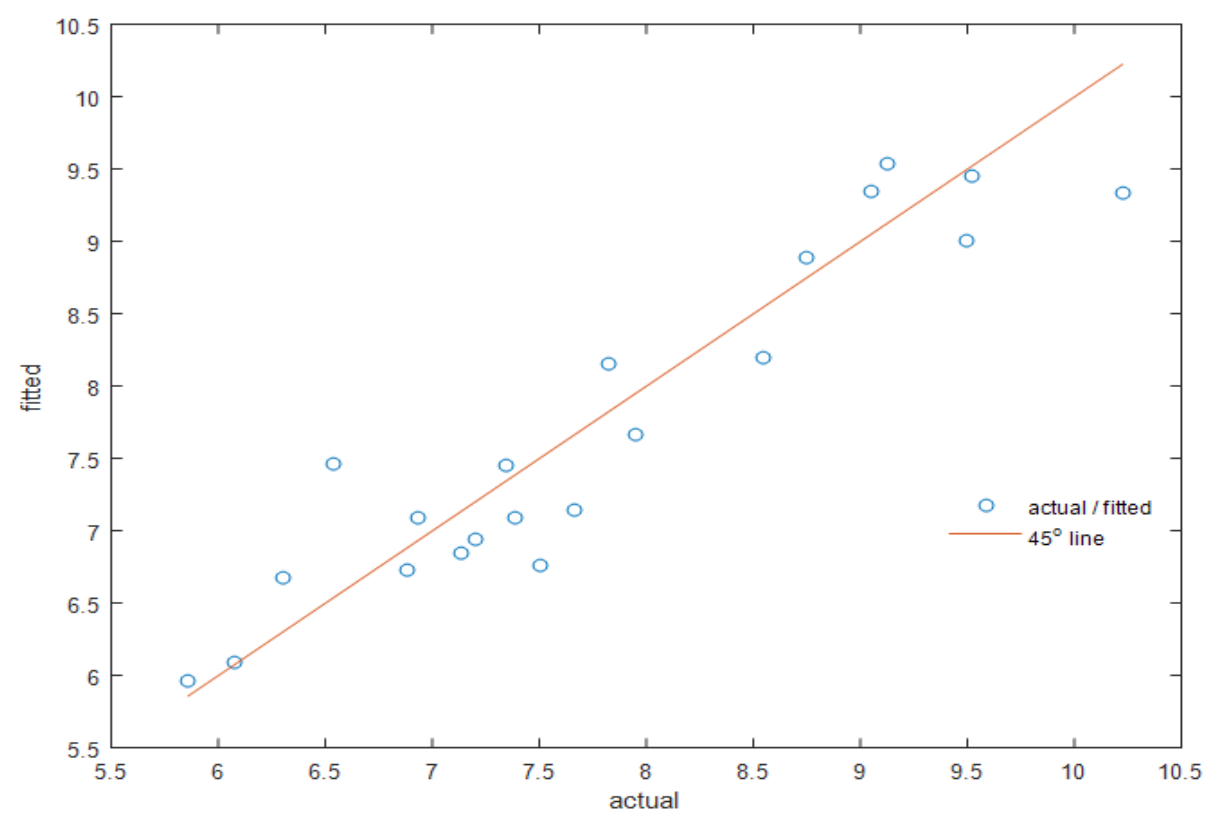

Figure 2. Elasticity of Advertising- Dynamic Model

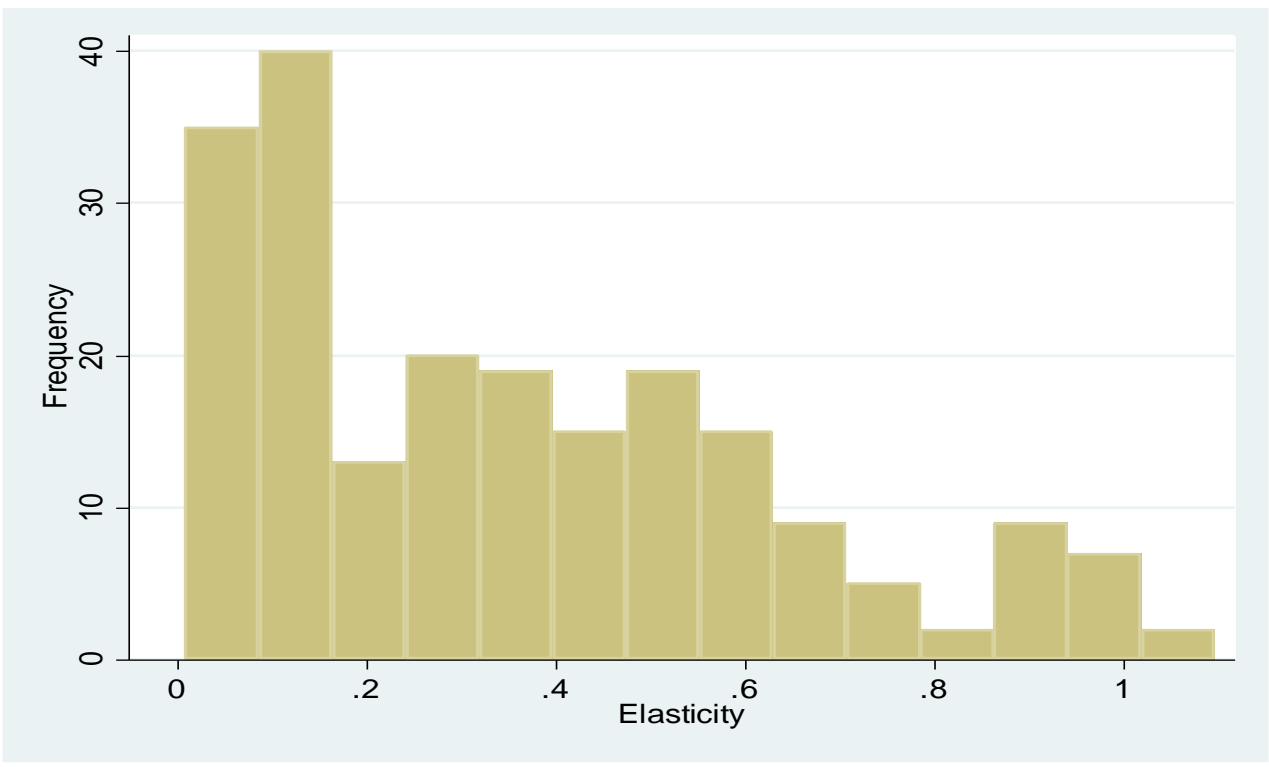




\section{References}

Ai, C., \& Norton, E. C. (2003). Interaction terms in logit and probit models. Economics letters, 80(1), 123-129.

Arellano, M., \& Bond, S. (1991). Some tests of specification for panel data: Monte Carlo evidence and an application to employment equations. The Review of Economic Studies, 58(2), 277-297.

Dumitrescu, E.-I. and Hurlin, C. (2012). Testing for Granger non-causality in heterogeneous panels. Economic Modelling, 29(4):1450-1460.

Fang, B., Ye, Q., \& Law, R. (2016). Effect of sharing economy on tourism industry employment. Annals of Tourism Research, 57, 264-267.

Greene, W. (1999). Marginal effects in the censored regression model. Economics Letters, 64(1), 43-49.

Hillman, A. J., \& Keim, G. D. (2001). Shareholder value, stakeholder management, and social issues: What's the bottom line? Strategic Management Journal, 125-139.

Saha, S., \& Yap, G. (2014). The moderation effects of political instability and terrorism on tourism development: A cross-country panel analysis. Journal of Travel Research, 53(4), 509-521 


\section{APPENDIX A: OUT-OF-SAMPLE VALIDATION}

This as an example of a simple linear model $y=f\left(x_{1}, x_{2}, x_{3}\right)$ that we estimate using the first 160 observations of our data, and we forecast the remaining 50 observations the results are shown in the following Figure. Evidently, the model overestimates the dependent variable relative to the actual value, so it cannot be acceptable in any practical sense of the term as the deviations between actual and predicted values are, apparently, systematic.

Figure A.1. Actual and fitted values in out-of-sample validation for a poor fitting model

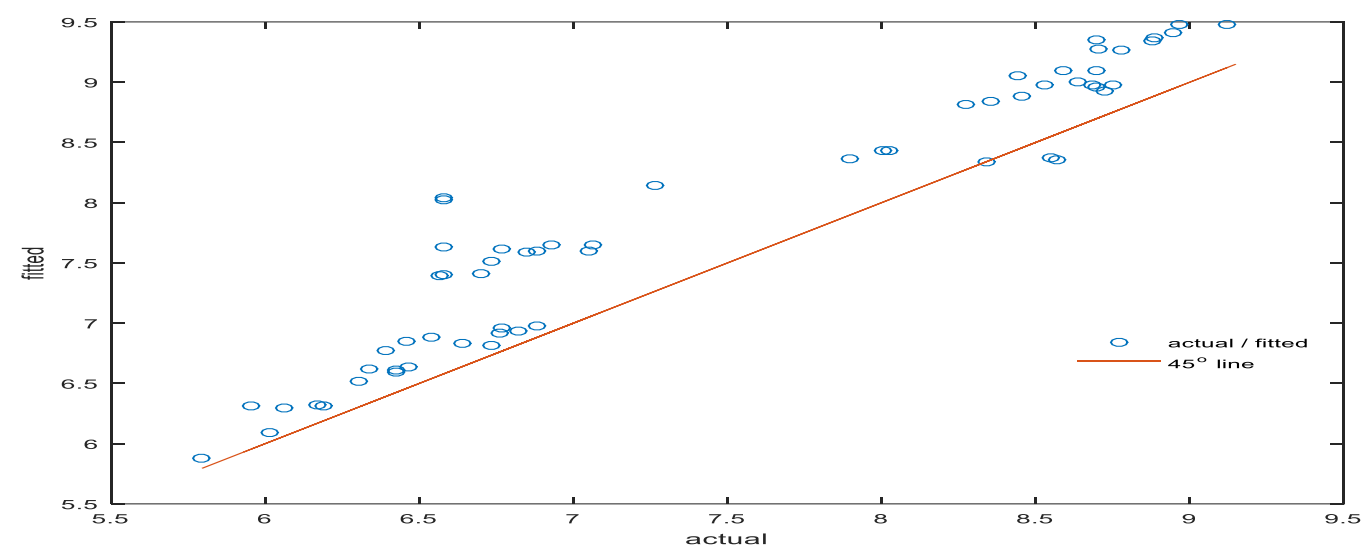

\section{APPENDIX B: SIMULATION EXPERIMENTS}

\section{Simulation experiment and specification tests}

In this Appendix we use artificial data to examine how diagnostic tests perform under various forms of misspecification. We use Monte Carlo to see how various diagnostics perform in this context. We tried the performance of diagnostic tests for both cross-sectional and panel contexts but we confine our attention here to panel data due to space limitation?. The main message is that most diagnostics perform well. For example, if an omitted variable is heteroscedastic, the heteroscedasticity tests are able to detect different variance of the error terms. RESET tests are able to detect problems when the omitted variable is a nonlinear function of the included variables. Autocorrelation tests detect a specification problem when the omitted variable is serially correlated.

\footnotetext{
${ }^{9}$ The results from the cross-sectional simulations can be obtained from the authors upon request.
} 
What is less known is how such tests perform when we have panel data where, additionally, we have to account for heterogeneity by using, for example, fixed effects. Diagnostic testing of panel data models is not well developed in econometrics and, certainly, such diagnostics are rarely if ever used in applied research. Our panel data model is:

$$
y_{i t}=\alpha_{i}+\beta_{1} x_{i t, 1}+\beta_{2} x_{i t, 2}+\beta_{3} x_{i t, 3}+u_{i t}, u_{i t} \sim i i d N\left(0,0.1^{2}\right), i=1, \ldots, n, t=1, \ldots, T,
$$

where the true values are $\beta_{1}=\beta_{2}=\beta_{3}=1$. We simply set $\alpha_{i}=i$. Moreover, $\mathrm{n}=20, \mathrm{~T}=5$, and

$$
x_{i t, 1} \sim \operatorname{iidN}(0,1), x_{i t, 2} \sim \operatorname{iidN}(0,1) .
$$

We consider an omitted variable which has stochastic heteroscedasticity of the following forms:

DGP 1: $x_{i t, 3} \sim N\left(0, \sigma_{i t}^{2}\right), \ln \sigma_{i t}^{2}=x_{i t, 1}+x_{i t, 2}+e_{i t}, e_{i t} \sim N\left(0,0.1^{2}\right)$

DGP 2: $x_{i t, 3} \sim N\left(0, \sigma_{i t}^{2}\right), \ln \sigma_{i t}^{2}=x_{i t, 1} x_{i t, 2}+e_{i t}, e_{i t} \sim N\left(0,0.1^{2}\right)$

DGP 3: $x_{i t, 3} \sim N\left(0, \sigma_{i t}^{2}\right), \ln \sigma_{i t}^{2}=x_{i t, 1}^{2}+x_{i t, 2}^{2}+e_{i t}, e_{i t} \sim N\left(0,0.1^{2}\right)$

DGP 4: $x_{i t, 3} \sim N\left(0, \sigma_{i t}^{2}\right), \ln \sigma_{i t}^{2}=\left|x_{i t, 1}\right|+\left|x_{i t, 2}\right|+e_{i t}, e_{i t} \sim N\left(0,0.1^{2}\right)$

DGP 5: $x_{i t, 3} \sim N\left(0, \sigma_{i t}^{2}\right), \ln \sigma_{i t}^{2}=\sin \left(x_{i t, 1}\right)+\cos \left(x_{i t, 2}\right)+e_{i t}, e_{i t} \sim N\left(0,0.1^{2}\right)$

The method of estimation is the standard fixed-effects estimator (also known as Least Squares with Dummy Variables, LSDV) and we apply the heteroscedasticity test to the squared residuals. The heteroscedasticity test, generally, is able to detect the misspecification but the RESET test cannot as we see from Table A.1 below. For the heteroscedasticity test most p-values greater than $5 \%$ occur $13 \%$ of the time under DGP2. In DGP5 the test fails to detect a problem as $55.5 \%$ of the time, pvalues exceed $5 \%$. On the other hand, the RESET test has p-values in excess of $5 \%$ almost all the time and only in DGP3 the exceedance occurs $27.3 \%$ of the time.

Table A.1. Heteroscedasticity and RESET Tests

\begin{tabular}{|l|c|c|}
\hline & Heteroscedasticity test & RESET test \\
\hline & p-values of F-test greater than 0.05 & p-values of F-test greater than 0.05 \\
\hline DGP 1 & $2.30 \%$ & $95.5 \%$ \\
\hline DGP 2 & 13.0 & 27.30 \\
\hline DGP 3 & 0.00 & 97.80 \\
\hline DGP 4 & 10.90 & 100 \\
\hline DGP 5 & 55.50 & \\
\hline
\end{tabular}


Next, we consider omitted variables related to $\mathrm{x}_{1}$ and $\mathrm{x}_{2}$ as follows:

DGP 1: $x_{i t, 3}=x_{i t, 1}^{2}+x_{i t, 2}^{2}+e_{i t}, e_{i t} \sim \operatorname{iidN}\left(0,0.1^{2}\right)$

DGP 2: $x_{i t, 3}=\left|x_{i t, 1}\right|+\left|x_{i t, 2}\right|+e_{i t}, e_{i t} \sim \operatorname{iidN}\left(0,0.1^{2}\right)$

DGP 3: $x_{i t, 3}=x_{i t, 1} x_{i t, 2}+e_{i t}, e_{i t} \sim i i d N\left(0,0.1^{2}\right)$

DGP 4: $x_{i t, 3}=\sin \left(x_{i t, 1}\right)+\cos \left(x_{i t, 2}\right)+e_{i t}, e_{i t} \sim i i d N\left(0,0.1^{2}\right)$

DGP 5: $x_{i t, 3}=\exp \left(x_{i t, 1}\right)+\exp \left(-x_{i t, 2}^{2}\right)+e_{i t}, e_{i t} \sim i i d N\left(0,0.1^{2}\right)$

DGP 6: $x_{i t, 3}=\left(x_{i t, 1}+x_{i t, 2}\right)^{2}+e_{i t}, e_{i t} \sim i i d N\left(0,0.1^{2}\right)$

The RESET test will fail to detect any problems, although the omitted variables are nonlinear functions of the regressors (details are omitted). As this is a significant problem, we propose to regress residuals on levels, squares and interactions of $\mathrm{x}_{1}$ and $\mathrm{x}_{2}$. This is equivalent to considering the fitted values but excluding the fixed effects. From Table A.2, the modified RESET test works now much better (and the heteroscedasticity test is also quite useful, as well). The p-values of the modified RESET test are almost never above 5\% even under DGP2 where the p-values of the heteroscedasticity test can be larger than $5 \%, 15.4 \%$ of the time, on average. This shows that the modified RESET test we use in this paper will perform well in panel data, revealing specification problems when excluded regressors are nonlinear functions of the included explanatory variables.

Table A.2. Heteroscedasticity, RESET and Modified RESET Tests

\begin{tabular}{|l|c|c|c|}
\hline & \multicolumn{2}{|l|}{ Heteroscedasticity test } & \multicolumn{2}{|l|}{ RESET test } \\
\hline & $\begin{array}{c}\text { p-values of F-test greater } \\
\text { than 0.05 }\end{array}$ & $\begin{array}{c}\text { p-values of F-test } \\
\text { greater than 0.05 }\end{array}$ & $\begin{array}{c}\text { p-values of F-test } \\
\text { greater than 0.05 }\end{array}$ \\
\hline DGP 1 & $0.000 \%$ & $96.40 \%$ & $0.200 \%$ \\
\hline DGP 2 & 15.40 & 99.00 & 0.200 \\
\hline DGP 3 & 0.000 & 97.10 & 0.300 \\
\hline DGP 4 & 0.300 & 98.10 & 0.497 \\
\hline DGP 5 & 0.000 & 67.30 & 0.200 \\
\hline DGP 6 & 0.000 & 88.90 & 0.000 \\
\hline
\end{tabular}

